\title{
Evaluation of the sedative and hypnotic effects of ethanol-extract isolated from Herba Agrimoniae in mice
}

\author{
Chang-lei $\mathrm{Li}^{1}$, Zhong-ze Lou ${ }^{2}$, Xiao-qing Chen ${ }^{1}$, Hui-ling $\mathrm{Yi}^{1}$, Meng-jia Chen ${ }^{3}$, \\ Bao-miao Ma ${ }^{4}{ }^{*}$ \\ ${ }^{1}$ School of Medicine, Jianghan University, Wuhan 430056, China \\ 2 Department of Psychosomatic Medicine, Ningbo First Hospital, Ningbo Hospital of Zhejiang \\ University, Ningbo 315010, China \\ ${ }^{3}$ Ningbo Urinary and Renal Diseases Hospital, Ningbo 315000, China \\ ${ }^{4}$ Wuhan Institutes of Biomedical Sciences, Jianghan University, Wuhan 430056, China \\ * Corresponding author. E-mail address: mabm.jhun@foxmail.com
}

\begin{abstract}
The purpose of this study was to evaluate the sedative and hypnotic activities of ethanol-extract isolated from Herba Agrimoniae on the central nervous system. Herba Agrimoniae has been used as a traditional herbal medicine for treating various cancers and diseases in traditional Chinese medicine. In the experiments, the extract of Herba Agrimoniae showed signifcant effects on mice, including reduced spontaneous activity, increased sleep ratio, shortened sleep latency and lengthened sleep time with a subthreshold or superthreshold dose of pentobarbital sodium. These findings confirmed that ethanol-extract isolated from Herba Agrimoniae had sedative and hypnotic effects and has potential to be preferable for the treatment of insomnia.
\end{abstract}

Keywords: Insomnia, Herba Agrimoniae, Sedative-hypnotic effects, Pentobarbital sleep

\section{Introduction}

Insomnia defined as persistent difficulty in falling or staying asleep that aflects daytime function, can induce significant psychological and physical disorder. Most patients engage in long-term use of benzodiazepines analogs to treat insomnia[1-3]. However, these drugs have limited benefits with significant side-effects, such as impaired cognitive function, memory and paradoxical effects such as aggression or behavioral disinhibition occasionally occur[4, 5]. In addition, long-term use is controversial because of concerns about adverse psychological and physical effects, decreasing effectiveness, and physical dependence and withdrawal[6, 7]. The search for novel herbal constituents from medicinal plants may provide better candidates for pharmacotherapeutics.

Herba Agrimoniae is a well known traditional medicinal plant with diverse properties including anti-oxidant, anti-inflammatory, analgesic and anti-tumor activity[8-10].The pharmacological activity of Herba Agrimoniae may primarily attributed to phenolic compounds such as agrimonin, catechin, querectin and rutin[11, 12]. The research on Herba Agrimoniae has focused mainly on the prevent or treat various diseases such as abdominal pain, sore throat, headaches, bloody discharge, parasitic infections and eczema [9, 13, 14]. Many studies have indicated that Herba Agrimoniae ethanol-extract can decrease blood glucose for alloxan-induced diabetic mice, and also showed that significant hypoglycemic effect for diabetic model mice whom caused by glucose, adrenaline and streptozotocin[15]. However, the effects of ethanol-extract isolated from Herba Agrimoniae on the central nervous system has not been revealed. Therefore, the present work was to investigate the effects of ethanol isolated of Herba Agrimonia on locomotion activity, pentobarbital-induced sleeping in mice. 


\section{Materials and methods}

\section{Animals}

Adult male Kunming mice (weighing $25 \pm 2 \mathrm{~g}$ ) were provided by the Animal Test Center of China Three Gorges University, license number: SCXK (E) 2017-0012. They were housed under controlled environment $\left(22 \pm 2^{\circ} \mathrm{C}, 12 \mathrm{~h}\right.$ light/dark cycle, free access to food and water). After 7 days of acclimatization, all mice were randomly divided into different groups $(n=12)$. This study was approved by the Jianghan university experimental animal ethical review committee (license No 201705002, date: 9/30/2017), and the experimental procedures are in compliance with the National Institutesof Health Guide for Care and Use of Laboratory Animals (Publication no. 85-23, revised 1985).

\section{Drugs and materials}

Herba Agrimoniae were collected from a chinese medicine shop of wuhan in China according to the guidelines of the Chinese Pharmacopoeia (2010). Pentobarbital sodium was obtained from JingDu biological technology Co. Ltd. (Shanghai,China), diazepam (DZP) injection was provided by Jianghan University Hospital. All other chemicals, reagents and equipment were of the highest grade available.

\section{Isolation of Agrimony}

Take Agrimony $300 \mathrm{~g}$, set $5000 \mathrm{~mL}$ round bottom flask, add 90\% ethanol $3000 \mathrm{~mL}$ reflux extraction $2 \mathrm{~h}$, suction filtration, the residue plus $90 \%$ ethanol $2000 \mathrm{~mL}$ reflux extraction 2 times each $1 \mathrm{~h}$,The filtrate was filtered, the filtrate was combined, ethanol was recovered, concentrated to a concentration of $1 \mathrm{~g}$ crude drug/ $\mathrm{mL}$, add an equal volume of pure water washed a total of three times to ethanol-free, dried in a vacuum oven and suspended at $400 \mathrm{~mL}$ water extract Herba Agrimoniae which be alternated. The dried extract was abbreviated as EIHA.

\section{Treatments}

For intraperitoneal (i.p.) injection, sodium pentobarbital was dissolved in physiological saline. The present study used $60 \mathrm{mg} / \mathrm{kg}$ (i.p.) as the hypnotic dose of sodium pentobarbital(with a 100\% rate of sleep onset) and $45 \mathrm{mg} / \mathrm{kg}$ (i.p.) as the subhypnotic dose (with a $0-10 \%$ rate of sleep onset). EIHA (3, 6 and $12 \mathrm{~g} / \mathrm{kg}$ ) and DZP $(2 \mathrm{mg} / \mathrm{kg}$ ) were dissolved with $1 \%$ dimethyl sulfoxide (DMSO) and administered intragastric(i.g.) $30 \mathrm{~min}$ before sodium pentobarbital administration (i.p.).

\section{Inner open-field behavior test}

The sedative activity was investigated by determining the spontaneous locomotor activity of mice in an open field. Animals were placed in an open filed experimental video analysis system, and acclimated to the activity cages individually for $5 \mathrm{~min}$. EIHA and DZP were administered orally $(0.1 \mathrm{ml} / 10 \mathrm{~g}$, volume/body weight $) 30 \mathrm{~min}$ before the test. The numbers of locomotion activity was counted for $5 \mathrm{~min}$.

\section{Pentobarbital-induced sleep test}

EIHA and DZP were administered intragastric(i.g.) $30 \mathrm{~min}$ before the pentobarbital (i.p.) injection. Following sodium pentobarbital injection, each mouse was observed for the onset of sleep. When the mice lost the righting reflex for over $1 \mathrm{~min}$, they were considered to be asleep. The loss of righting reflex was defined as a failure of the mouse to right itself for at least $10 \mathrm{~s}$ after being placed on its back.Time elapsed between the administration of sodium pentobarbital and sleep latency was recorded as the time of pentobarbital injection to the time of sleep onset, and sleep duration was defined as the elapsed time between the righting reflex loss and recovery. In the subhypnotic dosage of sodium pentobarbital treatment test, the percentage of sleep onset was calculated as follows: sleep onset $(\%)=$ no. falling asleep/total no. $\times 100$.

\section{Statistical analysis}


All data are presented as the mean \pm S.E.M. For statistical comparisons, the results were analyzed by a one-way analysis of variance (ANOVA) followed by the Students-Newman-Keuls test (SNK) for post-hoc comparisons. For the subhypnotic dosage of pentobarbital test, a chi-square test was used to compare the number of mice that fell asleep. Differences with $\mathrm{P}<0.05$ were considered statistically significant.

\section{Results}

\section{Effects on spontaneous activity of mice}

The sedative activity of EIHA was investigated by the locomotion test of mice. EIHA $(12 \mathrm{~g} / \mathrm{kg})$ reduced the locomotion activity of mice dose from $231.00 \pm 7.61$ to $195.06 \pm 6.67(\mathrm{P}<0.05)$ (Fig. 1). Positive control DZP $(2 \mathrm{mg} / \mathrm{kg})$ also significantly decreased the locomotion activity of mice $(\mathrm{P}<$ $0.01)$.

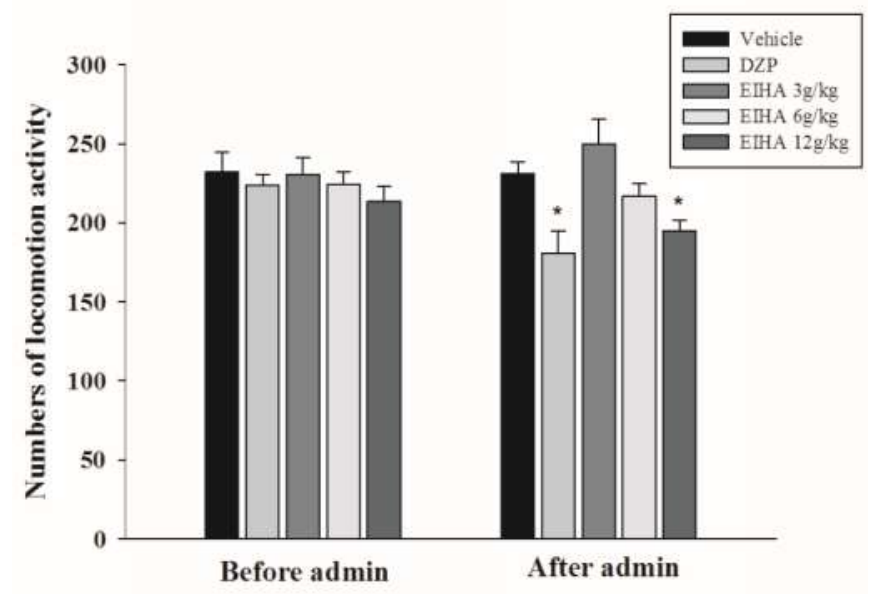

Fig. 1. Inhibitory effect of EIHA on spontaneous locomotor activity in mice. Five minutes after administration of DZP (2 mg/kg, i.g.) and EIHA (3, 6 and $12 \mathrm{~g} / \mathrm{kg}$, i.g.), spontaneous locomotor activity is measured. Each column represents the mean \pm S.E.M $(n=12) . * P<0.05$ compared with the vehicle group.

\section{Influence on the sedative effect with subthreshold dose of pentobarbital sodium}

As shown in Table 2, EIHA(12 g/kg, i.g.) pretreatment significantly increased the rate of sleep onset with a subhypnotic dose of pentobarbital $(40 \mathrm{mg} / \mathrm{kg}$, i.p.) compared with that of the vehicle group.

Table 2. The effect of EIHA on the sleep onset of mice treated with a subhypnotic dose of sodium pentobarbital.

\begin{tabular}{|c|c|c|c|}
\hline Group & Dosage & No. of falling asleep/total & Sleep onset (\%) \\
\hline Vehicle & - & $12 / 12$ & 0 \\
\hline \multirow{2}{*}{ DZP } & $2 \mathrm{mg} / \mathrm{kg}$ & $11 / 12$ & $91.7^{*}$ \\
\hline \multirow{3}{*}{ EIHA } & $3 \mathrm{~g} / \mathrm{kg}$ & $3 / 12$ & 25 \\
\cline { 2 - 4 } & $6 \mathrm{~g} / \mathrm{kg}$ & $4 / 12$ & 33.3 \\
\cline { 2 - 4 } & $12 \mathrm{~g} / \mathrm{kg}$ & $8 / 12$ & $66.7^{*}$ \\
\hline
\end{tabular}

Thirty minutes after administration of DMSO, DZP, and EIHA, sodium pentobarbital $(40 \mathrm{mg} / \mathrm{kg}$, i.p.) was given to mice. The number of mice falling asleep was recorded $(\mathrm{n}=12) .{ }^{*} \mathrm{P}<0.01$, compared with the vehicle group, chi-square test. 
In mice treated with a hypnotic dose of pentobarbital $(60 \mathrm{mg} / \mathrm{kg}$, i.p. $)$, EIHA significantly potentiated the hypnotic effects of pentobarbital by reducing sleep latency at the dosages of $12 \mathrm{~g} / \mathrm{kg}$ $(\mathrm{P}<0.05)$ (Fig. 3A) and prolonged sleep duration at the dosages of $12 \mathrm{~g} / \mathrm{kg}(\mathrm{P}<0.05)$ (Fig. 3B).

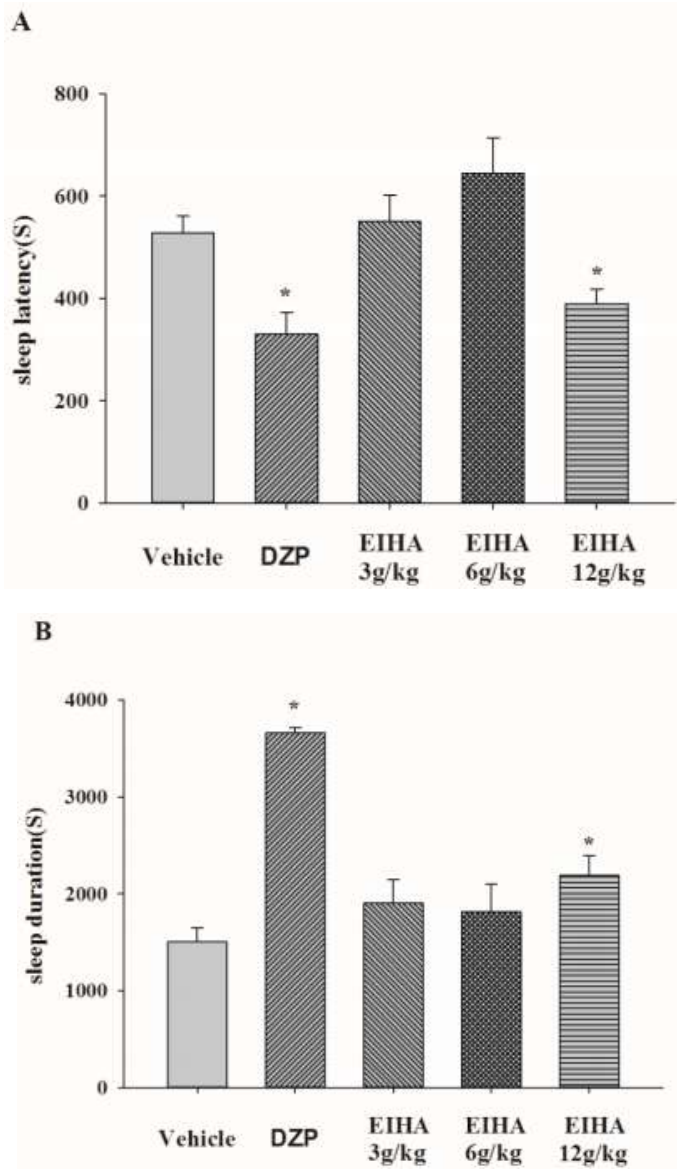

Fig. 3. Effect of EIHA on the hypnotic response to pentobarbital-induced sleep in mice. The sleep latency (A) and the sleep duration (B) are assessed. All data are presented as mean \pm S.E.M ( $\mathrm{n}=12)$, $* \mathrm{P}<0.05$ compared with the vehicle group.

\section{Conclusion}

In conclusion, this work represents the EIHA had sedative, hypnotic actions. But the mechanism of regulating sleep of EIHA is not clear. Further investigation will be necessary for active compounds and extensional mechanism.

\section{Acknowledgements}

This research was financially supported by the Nature Science Foundation of Ningbo (2016A610156); the Medicine and Health Science and Technology Plan Project in Zhejiang Province (2018KY671)

\section{References}

[1] Ashton H. The diagnosis and management of benzodiazepine dependence. Curr Opin Psychiatry. 2005. 18(3): 249-55. 
[2] Nenasteva AY. [Phenazepam: to the question of the efficacy and safety of benzodiazepine derivatives]. Zh Nevrol Psikhiatr Im S S Korsakova. 2017. 117(12): 156-161.

[3] Riemann D, Baglioni C, Bassetti C, et al. European guideline for the diagnosis and treatment of insomnia. J Sleep Res. 2017. 26(6): 675-700.

[4] Roth T, Drake C. Evolution of insomnia: current status and future direction. Sleep Med. 2004. 5 Suppl 1: S23-30.

[5] Bushnell GA, Stürmer T, Gaynes BN, Pate V, Miller M. Simultaneous Antidepressant and Benzodiazepine New Use and Subsequent Long-term Benzodiazepine Use in Adults With Depression, United States, 2001-2014. JAMA Psychiatry. 2017. 74(7): 747-755.

[6] Lader M, Tylee A, Donoghue J. Withdrawing benzodiazepines in primary care. CNS Drugs. 2009. 23(1): 19-34.

[7] Michelini S, Cassano GB, Frare F, Perugi G. Long-term use of benzodiazepines: tolerance, dependence and clinical problems in anxiety and mood disorders. Pharmacopsychiatry. 1996. 29(4): 127-34.

[8] Koshiura R, Miyamoto K, Ikeya Y, Taguchi H. Antitumor activity of methanol extract from roots of Agrimonia pilosa Ledeb. Jpn J Pharmacol. 1985. 38(1): 9-16.

[9] Wang JP, Hsu MF, Teng CM. Antihemostatic effect of Hsien-Ho-T'sao (Agrimonia pilosa). Am J Chin Med. 1984. 12(1-4): 116-23.

[10] Jung CH, Kim JH, Park S, Kweon DH, Kim SH, Ko SG. Inhibitory effect of Agrimonia pilosa Ledeb. on inflammation by suppression of iNOS and ROS production. Immunol Invest. 2010. 39(2): 159-70.

[11] Miyamoto K, Kishi N, Murayama T, Furukawa T, Koshiura R. Induction of cytotoxicity of peritoneal exudate cells by agrimoniin, a novel immunomodulatory tannin of Agrimonia pilosa Ledeb. Cancer Immunol Immunother. 1988. 27(1): 59-62.

[12]Xu X, Qi X, Wang W, Chen G. Separation and determination of flavonoids in Agrimonia pilosa Ledeb. by capillary electrophoresis with electrochemical detection. J Sep Sci. 2005. 28(7): 647-52.

[13]Liu WJ, Hou XQ, Chen H, Liang JY, Sun JB. Chemical constituents from Agrimonia pilosa Ledeb. and their chemotaxonomic significance. Nat Prod Res. 2016. 30(21): 2495-9.

[14]Park SH, Sim YB, Kang YJ, Lee JK, Lim SS, Suh HW. Effect of Agrimonia pilosa Ledeb Extract on the Antinociception and Mechanisms in Mouse. Korean J Physiol Pharmacol. 2012. 16(2): 119-23.

[15] Wang Y, Liu M, Zheng L, et al. Preparative purification of five bioactive components from Agrimonia pilosa Ledeb by high-speed counter-current chromatography. J Sep Sci. 2012. 35(15): 1977-84. 